\title{
Treatable traits in bronchiectasis
}

\author{
Rita Boaventura ${ }^{1}$, Oriol Sibila $\mathbb{1}^{2}$, Alvar Agusti $^{3}$ and James D. Chalmers ${ }^{4}$
}

Affiliations: ${ }^{1}$ Pulmonology Dept, Centro Hospitalar São João, Porto, Portugal. ${ }^{2}$ Respiratory Dept, Hospital de la Santa Creu i Sant Pau, Barcelona, Spain. ${ }^{3}$ Respiratory Institute, Hospital Clinic, University of Barcelona, IDIBAPS, CIBERES, Barcelona, Spain. ${ }^{4}$ Scottish Centre for Respiratory Research, University of Dundee, Dundee, UK.

Correspondence: James D. Chalmers, Scottish Centre for Respiratory Research, University of Dundee, Ninewells Hospital and Medical School, Dundee, DD1 9SY, UK. E-mail: jchalmersdadundee.ac.uk

@ERSpublications

A treatment approach based on "treatable traits" may provide better outcomes in the treatment of bronchiectasis http://ow.ly/ywaF30171OG

Cite this article as: Boaventura R, Sibila O, Agusti A, et al. Treatable traits in bronchiectasis. Eur Respir J 2018; 52: 1801269 [https://doi.org/10.1183/13993003.01269-2018].

The diagnostic label "bronchiectasis" describes the existence of localised and permanent airway dilation, but when used to describe a disease it includes a heterogeneous group of disorders that differ significantly in terms of aetiological, clinical, radiological, functional and microbial features [1]. Using cluster analysis, some previous studies have attempted to identify distinct "clinical phenotypes" in patients with bronchiectasis [2-4] (defined as "a single or combination of disease attributes that describe differences between patients that are related to clinically meaningful outcomes" [5]). By and large, however, these studies did not consider the underlying biology or response to therapy (i.e. the underlying endotype(s) [6]). Potential endotypes in bronchiectasis include immunodeficiency, ciliary dyskinesia, infection (with typical bacteria and non-tuberculous mycobacteria (NTM)), hypersensitivity to fungi and autoimmunity. Importantly, all of them can potentially become therapeutic targets [7].

The main treatment goals in bronchiectasis are to reduce symptoms, prevent exacerbations and lung function decline and, ultimately, improve survival. Unfortunately, most currently available therapeutic options have shown only a modest impact on disease outcomes in randomised clinical trials [8, 9]. For instance, inhaled antibiotic treatments have, so far, produced only modest benefits in terms of reduced exacerbations or improved quality of life, suggesting that endotypes other than airway "infection" are likely to play a relevant pathogenic role [10-12]. Likewise, co-existing airways diseases are common in patients with bronchiectasis and, in fact, up to $50 \%$ of these patients have a diagnosis of co-existing asthma or chronic obstructive pulmonary disease (COPD) [13, 14]. All in all, the complexity of bronchiectasis is poorly adapted to the "one size fits all" approach of current clinical guidelines. The co-existence in the same patient of different endotypes, clinical phenotypes and exposures requires a more precise approach to both assessment and therapy.

The concept of "treatable traits" was originally proposed in 2016 by AgUsTi et al. [15] in the European Respiratory Journal as a way toward precision medicine of airway diseases. These authors argued that the current airways disease diagnostic labels are imprecise, often overlap and lead to empirical therapy. They proposed that a biomarker-directed approach, based on the recognition of clinical phenotype and endotypes, can help to personalised treatment options which, hopefully, may result in better clinical outcomes. In the original manuscript on treatable traits, bronchiectasis was considered as a potential trait in patients with a diagnostic label of asthma or COPD. Here, we leverage from the treatable traits concept [15] and suggest that patients with bronchiectasis, with or without co-existing COPD and asthma, represent a heterogeneous group of patients who also present multiple treatable traits, many of which go 
TABLE 1 Proposed treatable traits of bronchiectasis separated into aetiological, pulmonary, non-pulmonary and environment/ lifestyle categories

Diagnostic criteria

Clinical features

Sputum characteristics

Inflammatory markers

Sputum culture

Chronic Pseudomonas infection

Mucus hypersecretion

Mucus plugging

Airflow obstruction

Asthma

Eosinophilia

NTM infection"

Aspergillus sensitisation

Bronchial hyperreactivity

Cough hypersensitivity

Respiratory insufficiency

Aetiological

Primary

immunodeficiencies

Cystic fibrosis

Primary ciliary

dyskinesia

ABPA $^{\pi}$

CTD
Two or more culture isolates at least 3 months apart in 1 year [35]

Volume

Colour of sputum

Clinical features

CT scan

FEV $1 / F V C<L L N$

Fixed ratio spirometry

GLI equations

Bronchodilator reversibility

Peak expiratory flow variability

Elevated sputum or blood eosinophils

Elevated sputum or blood eosinophils

Exclude other causes of eosinophilia

Positive culture and clinical/radiological findings

Elevated specific IgE/prick test positive

Challenge tests

Clinical features

Search other potential extrapulmonary causes

Capsaicin cough challenge

$\mathrm{PaO}_{2}<55 \mathrm{mmHg}$

$\mathrm{PaCO}_{2}>45 \mathrm{mmHg}$

Serum immunoglobulins levels

Specific antibody levels

Clinical features

Sweat chloride testing, CFTR genetic analysis and/or CFTR physiological testing

Clinical features ${ }^{+}$

Nasal NO assay

Electron microscopy ciliary structure analysis or video recording ciliary function analysis

Genetic testing $[42,43]$

Raised specific IgE and/or positive prick skin test to fungi, raised total IgE

Other: eosinophilia, radiological features, raised specific IgG/precipitating

antibodies against fungi [8]

Clinical features

Serum antibodies

Treatment

Expected benefits of treatment

Airway clearance $[33,34]$

Prompt treatment of exacerbations

Long term oral or inhaled antibiotics

Long term inhaled antibiotics

Long term macrolides

Airway clearance

Eradication at first isolation [8]

Airway clearance

Airway adjunct devices [27, 28]

Mucoactive drugs

Anti-inflammatories

Airway clearance

Mucoactive drugs

Nebulised saline

Anti-inflammatories

Bronchodilators

Smoking cessation

Exercise

ICS

Systemic corticosteroids

Bronchodilator

Leukotriene receptor antagonists

Monoclonal antibody anti-IL-5, anti-lgE [38]

ICS

Systemic corticosteroids

Treatment for underlying cause

Long term antibiotic [39]

ICS

Systemic corticosteroids

Antifungals

ICS

Antitussive

Chest physiotherapy

Long term oxygen and/or noninvasive ventilation

Reference to immunology specialist Immunoglobulin replacement

Reference to cystic fibrosis clinic CFTR modulators

DNAse

Genetic counselling

Intensive airway clearance

Management of upper airway

symptoms

Systemic corticosteroids and/or antifungals

Monoclonal antibody anti-IgE ICS

Reference to rheumatologist Immunosuppressors
Reduce exacerbations Improve quality of life

Reduce exacerbations Improve quality of life [36]

Slow lung function decline

Prevent chronic infection [37]

Reduce sputum volume

Reduce viscosity/increase ease of expectoration

Reduce sputum volume

Reduce viscosity/increase ease of expectoration

Improved exercise capacity and functional status [8]

Reduce exacerbations

Improve QoL and treatment response

Improve QoL and achieve remission $[40,41]$

Reduce exacerbations

Reduce sputum production Improved QoL [21]

Reduce exacerbations

Improve QoL

Improve quality of life Improve survival

Improve outcome Improve QoL

Prevent lung damage

Improve outcome

Improve QoL

Prevent lung damage

Improve outcome

Improve QoL

Prevent lung damage

Improve outcome

Improve QoL

Prevent lung damage

Improve outcome

Improve QoL

Prevent lung damage 


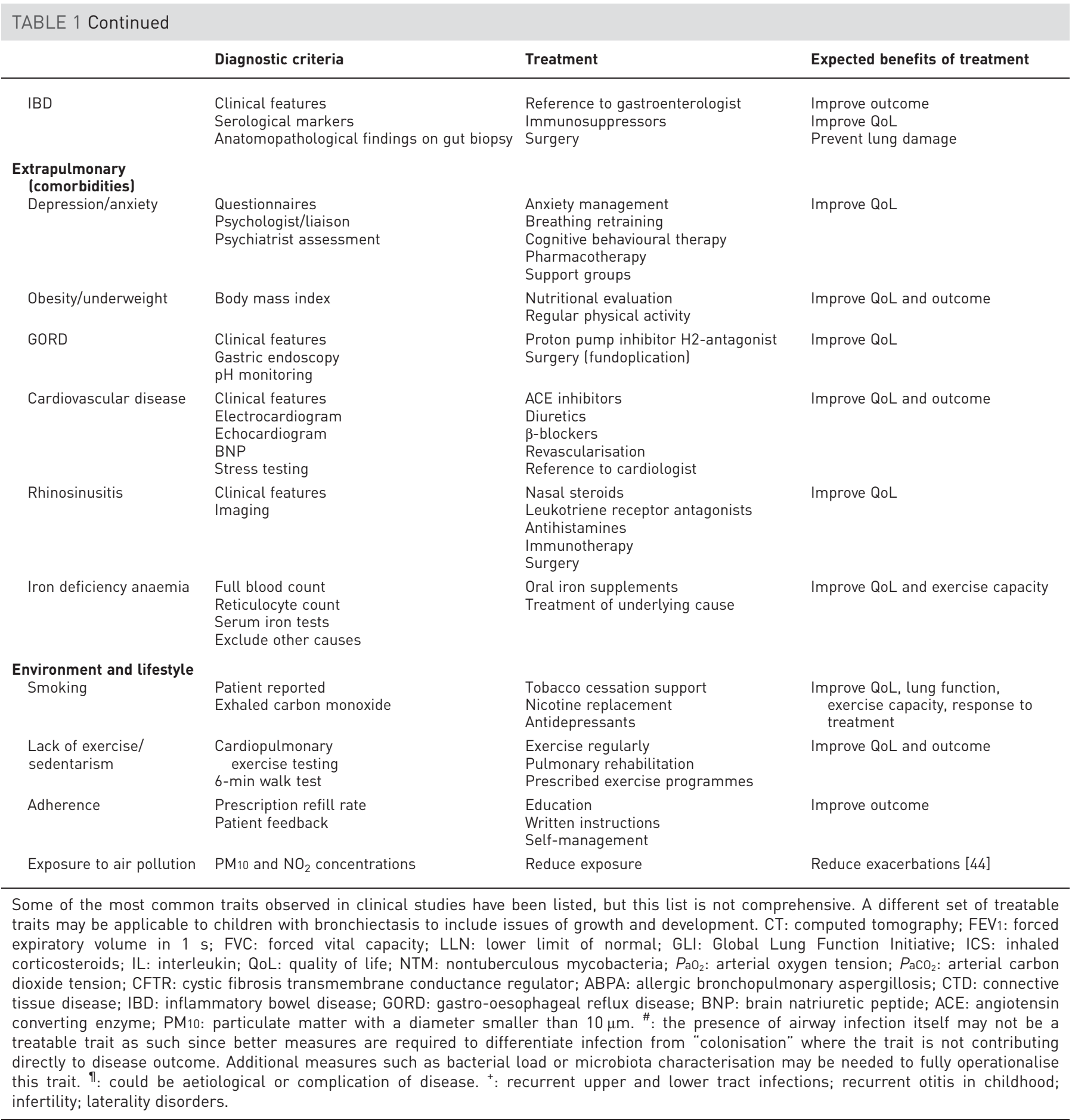

unrecognised (hence untreated) in clinical practice [16]. We venture that the application of the treatable traits approach to patients with bronchiectasis may, hopefully, contribute to a more personalised and precise management of these patients and, eventually, to improved clinical outcomes.

The treatable traits approach is attractive in bronchiectasis because it takes a "label free" approach to management. When faced with patients with "COPD-bronchiectasis overlap (BCO)" or "asthma-bronchiectasis overlap (ABO)", or patients with features of all three diseases (ABCO), physicians have been previously asked to consider which is the "predominant disorder" $[10,11,17-20]$. We argue here that this approach is flawed because the concept of overlap syndromes itself is flawed. COPD and asthma are complex and heterogeneous entities, as bronchiectasis is. Clarity is not created by generating another poorly defined clinical entity (e.g. $\mathrm{ACO}, \mathrm{BCO}, \mathrm{ABO}$ or $\mathrm{ABCO}$ ) $[13,17,18]$. An approach that is 
more user-friendly in terms of the underlying biology is to overlook diagnostic labels and focus instead on the pathophysiology (i.e. endotypes) and clinical phenotypes present in any given patient. This idea is the core of the treatable traits concept. For instance, the treatable traits concept applied to bronchiectasis should recognise that airway infection is only one of many treatable traits in a given patient and, therefore, allows us to understand why antibiotic treatment may not improve outcomes in an individual in whom symptoms or exacerbations are driven by a different treatable trait (e.g. upper airway disease, eosinophilia, fungal allergy or comorbidities) [21-24]. This strategy may also help in understanding why randomised clinical trials have shown inconsistent results despite similar design and well-defined cohorts. For instance, patients enrolled in the RESPIRE trials of inhaled dry powder ciprofloxacin had a history of two or more prior exacerbations and positive sputum cultures for respiratory bacteria. Despite apparently enriching for a "treatable trait" of airway infection, four trial arms provided inconsistent results [10-12]; similar inconsistency was seen in the AIRBX studies of aztreonam where two replicate trials with similar subjects showed an improvement in quality of life in one study and not in the other [25]. Likewise, inhaled dry powder mannitol failed to reduce frequency of exacerbations, despite the fact that it modestly improved quality of life [26]. The overall effect sizes in terms of reduced exacerbations or improved quality of life have been modest in bronchiectasis trials in general [10,11,25-28]. The presence of multiple competing

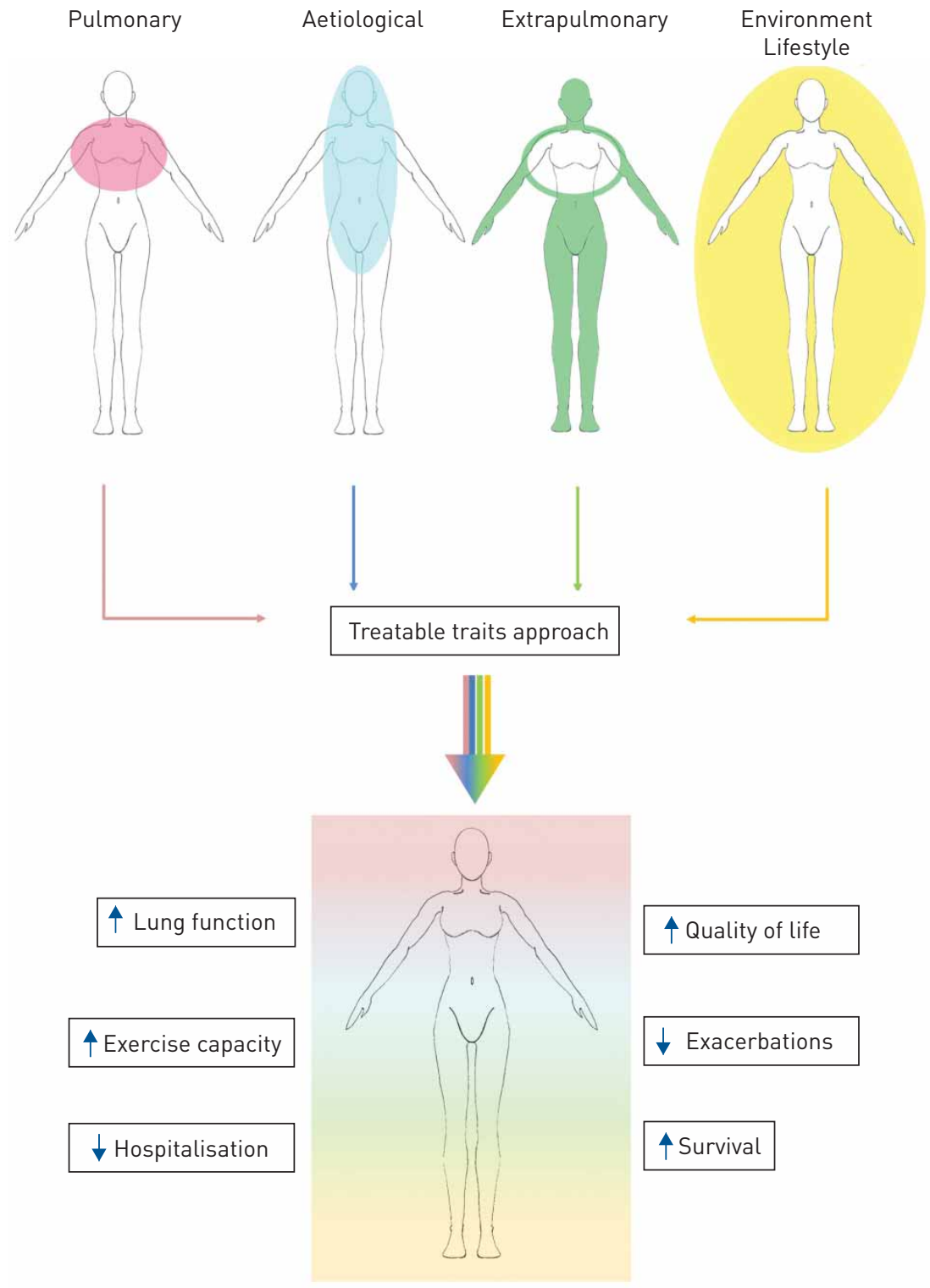

FIGURE 1 Using the treatable traits concept to improve treatment outcomes in bronchiectasis. Recognition and treatment of multiple pulmonary, aetiological, extrapulmonary and environmental/lifestyle associated traits should lead to more holistic treatment and a greater impact on relevant clinical outcomes. 
"treatable traits" within the population studied may contribute to explaining why treating a single trait (such as bronchial infection or airway dehydration) achieves only a modest reduction in exacerbations or improvement in symptoms.

The recently published European Respiratory Society (ERS) guidelines for the management of bronchiectasis advocate a number of treatments, including antibiotics, bronchodilators, pulmonary rehabilitation and chest physiotherapy [8]. While some guidance is provided on the optimal populations to benefit (e.g. based on prior exacerbation history), patient phenotyping or endotyping is beyond the scope of such a document. Likewise, the guideline cannot address treatable comorbidities, environmental, lifestyle and psychological factors, which can also impact on patient outcomes [2, 29-32]. Providing holistic care for patients with bronchiectasis requires taking these factors into consideration as well. Thus, recognising the limitations of existing approaches, we have applied the treatable traits model proposed by Agusti et al. [15] to bronchiectasis. Agusti et al. [15] divided potential treatable traits of airways disease into three broad categories (pulmonary, extrapulmonary, and behaviour and lifestyle treatable traits). We think that these same categories apply to bronchiectasis but we propose to add a fourth category for bronchiectasis that includes "aetiological" treatable traits to emphasise the importance of identifying those traits which directly lead to the development of bronchiectasis such as NTM infection, allergic bronchopulmonary aspergillosis and immunodeficiency. Table 1 shows a list of proposed treatable traits, diagnostic criteria, suggested therapies and expected benefits in patients with bronchiectasis. Crucially, this hypothesis should be prospectively tested or, alternatively, analysed retrospectively in randomised clinical trial datasets if pharmaceutical companies are willing to share their data.

In summary, we propose here that the treatable traits approach can be a useful strategy to help clinicians consider the many different aspects that must be addressed for the appropriate clinical management of patients with bronchiectasis. Focusing on a multimodality approach to treatment is likely to result in better clinical outcomes (figure 1) but this should ideally be tested in prospective studies. Identifying the frequency and clinical impact of individual treatable traits in bronchiectasis, as well as determining the best way to address them, will be important in developing this concept further. As suggested in the conclusions of a recent ERS research seminar, in order to prove that the treatable traits strategy is feasible, efficacious and safe in clinical practice, we need to formally test this hypothesis using the novel platform of adaptive trials design [45].

Conflict of interest: J.D. Chalmers reports grants for COPD research and personal fees from GlaxoSmithKline, Boehringer Ingelheim and Pfizer, grants for COPD research from AstraZeneca, grants for bronchiectasis research and personal fees from Bayer Healthcare and Grifols, and personal fees for consultancy from Napp, outside the submitted work.

\section{References}

1 Araujo D, Shteinberg M, Aliberti S, et al. Standardised classification of the aetiology of bronchiectasis using an objective algorithm. Eur Respir J 2017; 50: 1701289.

2 Chalmers JD, Aliberti S, Filonenko A, et al. Characterization of the "frequent exacerbator phenotype" in bronchiectasis. Am J Respir Crit Care Med 2018; 197: 1410-1420.

3 Aliberti S, Lonni S, Dore S, et al. Clinical phenotypes in adult patients with bronchiectasis. Eur Respir J 2016; 47 : $1113-1122$.

4 Martinez-Garcia MA, Vendrell M, Giron R, et al. The multiple faces of non-cystic fibrosis bronchiectasis A cluster analysis approach. Ann Am Thorac Soc 2016; 13: 1468-1475.

5 Han MK, Agusti A, Calverley PM, et al. Chronic obstructive pulmonary disease phenotypes: the future of COPD. Am J Respir Crit Care Med 2010; 182: 598-604

6 Anderson GP. Endotyping asthma: new insights into key pathogenic mechanisms in a complex, heterogeneous disease. Lancet 2008; 372: 1107-1119.

7 Chalmers JD, Chotirmall SH. Bronchiectasis: new therapies and new perspectives. Lancet Respir Med 2018; in press [https://doi.org/10.1016/S2213-2600(18)30053-5].

8 Polverino E, Goeminne PC, McDonnell MJ, et al. European Respiratory Society guidelines for the management of adult bronchiectasis. Eur Respir J 2017; 50: 1700629.

9 Aliberti S, Masefield S, Polverino E, et al. Research priorities in bronchiectasis: a consensus statement from the EMBARC Clinical Research Collaboration. Eur Respir J 2016; 48: 632-647.

10 De Soyza A, Aksamit T, Bandel T-J, et al. RESPIRE 1: a phase III placebo-controlled randomised trial of ciprofloxacin dry powder for inhalation in non-cystic fibrosis bronchiectasis. Eur Respir J 2018; 51: 1702052.

11 Aksamit T, De Soyza A, Bandel T-J, et al. RESPIRE 2: a phase III placebo-controlled randomised trial of ciprofloxacin dry powder for inhalation in non-cystic fibrosis bronchiectasis. Eur Respir J 2018; 51: 1702053.

12 Chotirmall SH, Chalmers JD. RESPIRE: breathing new life into bronchiectasis. Eur Respir J 2018; 51: 1702444.

13 Mao B, Yang J-W, Lu H-W, et al. Asthma and bronchiectasis exacerbation. Eur Respir J 2016; 47: 1680-1686.

14 Quint JK, Millett ERC, Joshi M, et al. Changes in the incidence, prevalence and mortality of bronchiectasis in the UK from 2004 to 2013: a population-based cohort study. Eur Respir J 2016; 47: 186-193.

15 Agusti A, Bel E, Thomas M, et al. Treatable traits: toward precision medicine of chronic airway diseases. Eur Respir J 2016; 47: 410-419. 
16 Aliberti S, Hill AT, Mantero M, et al. Quality standards for the management of bronchiectasis in Italy: a national audit. Eur Respir J 2016; 48: 244-248.

17 Vogelmeier CF, Criner GJ, Martinez FJ, et al. Global Strategy for the Diagnosis, Management, and Prevention of Chronic Obstructive Lung Disease 2017 Report: GOLD Executive Summary. Eur Respir J 2017; 49: 1700214.

18 Hurst JR, Elborn JS, De Soyza A. COPD-bronchiectasis overlap syndrome. Eur Respir J 2015; 45: 310-313.

19 Wedzicha JA, Miravitlles M, Hurst JR, et al. Management of COPD exacerbations: a European Respiratory Society/American Thoracic Society guideline. Eur Respir J 2017; 49: 1600791.

20 Sin DD, Miravitlles M, Mannino DM, et al. What is asthma-COPD overlap syndrome? Towards a consensus definition from a round table discussion. Eur Respir J 2016; 48: 664-673.

21 Agarwal R, Aggarwal AN, Dhooria S, et al. A randomised trial of glucocorticoids in acute-stage allergic bronchopulmonary aspergillosis complicating asthma. Eur Respir J 2016; 47: 490-498.

22 Saleh AD, Kwok B, Brown JS, et al. Correlates and assessment of excess cardiovascular risk in bronchiectasis. Eur Respir J 2017; 50: 1701127.

23 Kim VL, Coombs NA, Staples KJ, et al. Impact and associations of eosinophilic inflammation in COPD: analysis of the AERIS cohort. Eur Respir J 2017; 50: 1700853.

24 Mac Aogain M, Chandrasekaran R, Lim AYH, et al. Immunological corollary of the pulmonary mycobiome in bronchiectasis: the CAMEB study. Eur Respir J 2018; 52: 1800766.

25 Barker AF, O'Donnell AE, Flume P, et al. Aztreonam for inhalation solution in patients with non-cystic fibrosis bronchiectasis (AIR-BX1 and AIR-BX2): two randomised double-blind, placebo-controlled phase 3 trials. Lancet Respir Med 2014; 2: 738-749.

26 Bilton D, Tino G, Barker AF, et al. Inhaled mannitol for non-cystic fibrosis bronchiectasis: a randomised, controlled trial. Thorax 2014; 69: 1073-1079.

27 Munoz G, de Gracia J, Buxo M, et al. Long-term benefits of airway clearance in bronchiectasis: a randomised placebo-controlled trial. Eur Respir J 2018; 51: 1701926.

28 Wong C, Sullivan C, Jayaram L. ELTGOL airway clearance in bronchiectasis: laying the bricks of evidence. Eur Respir J 2018; 51: 1702232.

29 McDonnell MJ, Aliberti S, Goeminne PC, et al. Comorbidities and the risk of mortality in patients with bronchiectasis: an international multicentre cohort study. Lancet Respir Med 2016; 4: 969-979.

30 Chalmers JD, Ringshausen FC, Harris B, et al. Cross-infection risk in patients with bronchiectasis: a position statement from the European Bronchiectasis Network (EMBARC), EMBARC/ELF patient advisory group and European Reference Network (ERN-Lung) Bronchiectasis Network. Eur Respir J 2018; 51: 1701937.

31 Goeminne PC, De Soyza A. Bronchiectasis: how to be an orphan with many parents? Eur Respir J 2016; 47: $10-13$.

32 Hill AT, Haworth CS, Aliberti S, et al. Pulmonary exacerbation in adults with bronchiectasis: a consensus definition for clinical research. Eur Respir J 2017; 49: 1700051.

33 Lee AL, Burge AT, Holland AE. Airway clearance techniques for bronchiectasis. Cochrane Database Syst Rev 2015; 11: CD008351

34 Lee AL, Burge AT, Holland AE. Positive expiratory pressure therapy versus other airway clearance techniques for bronchiectasis. Cochrane Database Syst Rev 2017; 9: CD011699.

35 Finch S, McDonnell MJ, Abo-Leyah H, et al. A comprehensive analysis of the impact of Pseudomonas aeruginosa colonization on prognosis in adult bronchiectasis. Ann Am Thorac Soc 2015; 12: 1602-1611.

36 Araujo D, Shteinberg M, Aliberti S, et al. The independent contribution of Pseudomonas aeruginosa infection to long-term clinical outcomes in bronchiectasis. Eur Respir J 2018; 51: 1701953.

37 Hilliam Y, Moore MP, Lamont IL, et al. Pseudomonas aeruginosa adaptation and diversification in the non-cystic fibrosis bronchiectasis lung. Eur Respir J 2017; 49: 1602108.

38 Humbert M, Taille C, Mala L, et al. Omalizumab effectiveness in patients with severe allergic asthma according to blood eosinophil count: the STELLAIR study. Eur Respir J 2018; 51: 1702523.

39 Griffith DE, Aksamit T, Brown-Elliott BA, et al. An official ATS/IDSA statement: diagnosis, treatment, and prevention of nontuberculous mycobacterial diseases. Am J Respir Crit Care Med 2007; 175: 367-416.

40 van Ingen J, Aksamit $\mathrm{T}$, Andrejak $\mathrm{C}$, et al. Treatment outcome definitions in nontuberculous mycobacteria pulmonary disease: an NTM-NET consensus statement. Eur Respir J 2018; 51: 1800170.

41 van Ingen $\mathrm{J}$, Wagner $\mathrm{D}$, Gallagher $\mathrm{J}$, et al. Poor adherence to management guidelines in nontuberculous mycobacterial pulmonary diseases. Eur Respir J 2017; 49: 1601855.

42 Lucas JS, Barbato A, Collins SA, et al. European Respiratory Society guidelines for the diagnosis of primary ciliary dyskinesia. Eur Respir J 2017; 49: 1601090.

43 Behan L, Dimitrov BD, Kuehni CE, et al. PICADAR: a diagnostic predictive tool for primary ciliary dyskinesia. Eur Respir J 2016; 47: 1103-1112.

44 Goeminne PC, Cox B, Finch S, et al. The impact of acute air pollution fluctuations on bronchiectasis pulmonary exacerbation: a case-crossover analysis. Eur Respir J 2018; 52: 1702557.

45 Agustí A, Bafadhel M, Beasley R, et al. Precision medicine in airway diseases: moving to clinical practice. Eur Respir J 2017; 50: 1701655. 